\title{
Pharmacovigilance: The Least Understood and Least Practiced Science in Dentistry
}

\author{
Chaya Chhabra ${ }^{1}$, Kumar G Chhabra ${ }^{2}$, Ashish Sharma ${ }^{3}$, Akanksha Goyal ${ }^{4}$, Gururaghavendran Rajesh ${ }^{5}$, Almas Binnal ${ }^{6}$
}

\begin{abstract}
Aim: The purpose of this review was to explore the overall scenario of pharmacovigilance, its aims, challenges, and recommendations pertaining to dentistry for further improvements.

Materials and methods: Literature search was done with the help of the Endnote software, followed by vigilantly arranging the material in a coordinated way.

Results: Adverse drug reactions (ADRs) are among the top 10 leading causes of all-cause mortality. In order to reduce the harm to the patients and serve the public health mission, there is a definite need for developing mechanisms for evaluating and monitoring the safety of medicines in India. Therefore, the requirement for developing a well-organized pharmacovigilance system is imperative. Different countries have developed their own reporting guidelines for pharmacovigilance. The reporting guidelines are conceived to adapt to the specific requirements of any country and the prime focus of these adverse event reporting systems is improving the patient's health and safety. The Central Drugs Standard Control Organization (CDSCO) has initiated a nation-wide pharmacovigilance program in India. It aims to protect the health of the patients by assuring drug safety under the aegis of Directorate General of Health Services, Ministry of Health and Family Welfare, Government of India. In dentistry, the science of pharmacovigilance is rarely applied and its practice among dental health professionals, including reporting of ADRs, is minimal. However, studies have shown that the dental professionals comprehend the importance of pharmacovigilance and its application in dentistry. Conclusion: It is essential to foster a sense of trust among patients regarding the medicines they use, to ensure that risks in drug use are anticipated and managed. This will eventually enhance the confidence of patients on the healthcare delivery system in general.

Clinical significance: The practice of dentistry involves prescribing various medications for patient use. It is therefore the duty of the dentist to comprehend and identify the adverse effects of the drugs. Dentists should also know about the right channel for reporting any instances of ADRs. They should also strive toward increasing the awareness among patients regarding possible side effects so that any untoward consequences can be avoided. This will be an invaluable aid in furthering the public health mission of improving the health of the populations.
\end{abstract}

Keywords: Dental professionals, Indian scenario, Pharmacovigilance.

World Journal of Dentistry (2019): 10.5005/jp-journals-10015-1661

\section{INTRODUCTION}

The major cause of death and socioeconomic disruption globally is various diseases afflicting mankind. In recent times, many diseases have emerged that may not have effective control or preventive measures. Hence need of the hour is the availability of newer, better, and safe drugs to tackle these newer morbidities. The manner in which the diseases are managed and controlled has drastically changed with the advent of modern drug systems.

However, the incidence of adverse drug reactions (ADRs) cannot be entirely ruled out. ${ }^{1}$ Adverse drug reaction has now emerged as one of the leading causes of all mortalities. The harm done by the drugs can be reduced by developing proper evaluation and monitoring methods for safety of the patients. This will be an invaluable aid in furthering the public health mission of improving the health of the populations. This can be carried effectively by developing a well-organized pharmacovigilance system. ${ }^{2}$ According to the World Health Organization (WHO) collaborating center for International Drug Monitoring, "Pharmacovigilance is the science and activities relating to the detection, assessment, understanding and prevention of adverse effects or any other possible drug-related problems." ${ }^{\prime 3}$

Pharmacovigilance begins at clinical trials and continues all long the product life. It can be broadly divided into before-marketing (premarketing) and after-marketing phases (postmarketing).
${ }^{1}$ Department of Pedodontics and Preventive Dentistry, MM College of Dental Sciences and Research, Ambala, Haryana, India

${ }^{2}$ Department of Public Health Dentistry, MM College of Dental Sciences and Research, Ambala, Haryana, India

${ }^{3}$ Department of Public Health Dentistry, RR Dental College and Hospital, Udaipur, Rajasthan, India

${ }^{4}$ Department of Home Science, University of Rajasthan, Jaipur, Rajasthan, India

${ }^{5}$ Department of Public Health Dentistry, Manipal College of Dental Sciences, Mangaluru, Manipal Academy of Higher Education, Manipal, Karnataka, India

${ }^{6}$ Department of Oral Medicine and Radiology, Manipal College of Dental Sciences, Mangaluru, Manipal Academy of Higher Education, Manipal, Karnataka, India

Corresponding Author: Kumar G Chhabra, Department of Public Health Dentistry, MM College of Dental Sciences and Research, Ambala, Haryana, India, Phone: +91-75970-43999, e-mail: rajsushil. chhabra@gmail.com

How to cite this article: Chhabra C, Chhabra KG, Sharma A, et al. Pharmacovigilance: The Least Understood and Least Practiced Science in Dentistry. World J Dent 2019;10(5):402-406.

Source of support: Nil

Conflict of interest: None

(C) The Author(s). 2019 Open Access This article is distributed under the terms of the Creative Commons Attribution 4.0 International License (https://creativecommons. org/licenses/by-nc/4.0/), which permits unrestricted use, distribution, and non-commercial reproduction in any medium, provided you give appropriate credit to the original author(s) and the source, provide a link to the Creative Commons license, and indicate if changes were made. The Creative Commons Public Domain Dedication waiver (http://creativecommons.org/publicdomain/zero/1.0/) applies to the data made available in this article, unless otherwise stated. 
The phase 1 of the clinical trial begins by collecting the information pertaining to the safety of the drug before the drug is approved. Studies will continue even after the release of the drug into the market. Postmarketing surveillance is now rendered mandatory by various drug regularity agencies around the world. ${ }^{4}$ Pharmacovigilance also deals with the following:

- Issues related to substandard medicines

- Errors in medication

- Lack of efficient reports

- Inappropriate prescription of medications when it is not indicated

- Reports of cases for poisoning, both acute and chronic

- Cases of mortality due to drug use

- Misuse and abuse of medicines

- Adverse interactions with chemicals, food, and other medicines.

\section{Aims of Pharmacovigilance 4}

- It aims to improvise the care given for the patients and their safety in relation to medicines and paramedical intervention

- It aims for improvement in safety of public health in relation to the drugs used by the public

- It aims in assessing the harm, risks, effectiveness, and benefits of the drugs and evaluating their safety, rational, and more effective use

- It aims to improve the knowledge, promote education, understanding, clinical training, and its communication to the public in pharmacovigilance.

\section{Global Perspectives of \\ Pharmacovigilance and ADR Reporting ${ }^{4}$}

Pharmacovigilance guidelines are not uniform across the world and show considerable variation from country to country. Reporting guidelines are based on requirements that are specific for a nation and have evolved to form a system addressing the needs of that nation. The main aim of the reporting system is to monitor the harmful effects of the drugs, which helps in improving the health of the patients.

A program under the WHO termed International Drug Monitoring was initiated in 1978 , with only 10 countries participating in it. It has now grown by leaps and bounds at the global level, with the active participation of 120 countries.

\section{Functions of National Pharmacovigilance SYSTEM ${ }^{5-7}$}

Following WHO's suggestions in January 2010 and also considering review of the Advisory Committee regarding Safety of Medical Product (ACSoMP) in April 2010, the National Pharmacovigilance System has adopted specific functions. These functions are as follows:

- Promotion of pharmacovigilance in a country has to be implemented by collecting the ADR reports along with substandard drugs and reports of medical errors

- The information should be collected from existing ADRs of the country and also cohorts that monitor internationally in defined patients or populations. This information has to be collaborated and harmonized for further considerations
- Drug safety indications pertaining to unknown and poorly characterized adverse events and other events that occur due to combination of drugs have to be identified

- The assessment of risk of ADR and outlining its management is to be undertaken

- The recognition of the quality of the medicine and endorsing it, also to identify the resulting ADRs that may occur due to the drug

- The team should also take responsibility for the effective communication on drug safety and also dispel the rumors of the toxicity of medicines and vaccines

- The information from pharmacovigilance has to be applied appropriately as it benefits individual patients, national medicine policies, public health programs, and treatment guidelines

- The development and maintenance of information related to drug utilization has to be undertaken

- The issues regarding unregulated prescribing of medicines and their dispensing are to be identified.

\section{Pharmacovigilance Structure in India ${ }^{8}$}

A nationwide pharmacovigilance program to protect the health of the patients was initiated by the Central Drugs Standard Control Organization (CDSCO). As a part of this program, the safety of drugs is assured under Directorate General of Health Services, Government of India, and Ministry of Health and Family Welfare, Government of India. Suggestions put forth by the WHO in the document titled "Safety Monitoring of Medicinal Products: Guidelines for Setting up and Running a Pharmacovigilance Center" were implemented. The Indian Pharmacopoeia Commission based in Ghaziabad functions as the National Coordinating Center (NCC) and it synchronizes the program under supervision of a steering committee. The National Pharmacovigilance Program was officially inaugurated on 23 November 2003 in New Delhi. Offices were established at regional and peripheral, zonal, and national levels to synchronize various activities pertaining to pharmacovigilance in India (Fig. 1).

\section{Broad Objectives of the Program}

- To develop the culture of reporting and notification of unfavorable events

- To establish a robust and broader ADR monitoring system in India.

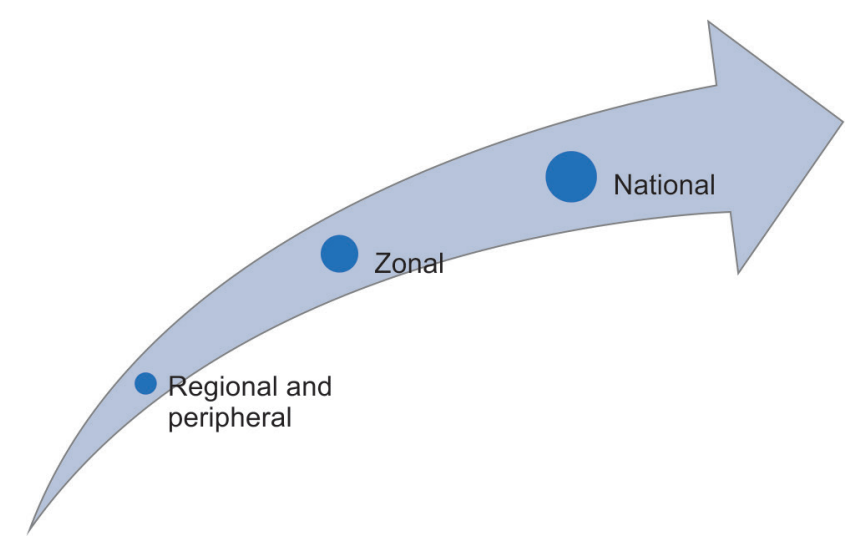

Fig. 1: Hierarchical structure of the pharmacovigilance centers in India 


\section{Discrete Objectives of the Program}

- To make an ADR database for the population of India

- To create awareness regarding observation of ADRs among general people

- To guard the safety of drugs in India

- Development of infrastructure for the regulatory reviews of periodic safety update reports (PSURs).

\section{National Pharmacovigilance Center ${ }^{9}$}

The National Pharmacovigilance Center is at CDSCO and performs the following activities:

- To ascertain the harmful drug reactions of the medicines that should be identified beforehand or to indicate certain commonly occurring reactions

- To always maintain a record of the periodic safety update reports (PSURs) provided by the pharmaceutical companies

- To exchange information on the drug safety and to establish contacts with the international organizations and agencies of pharmacovigilance

- To promulgate safety regulations outlining the actions to be taken, if necessary, to improve the safe use of the drug

- To disseminate the information on the adverse effects of drugs through bulletins, alerts, news, and seminars.

\section{National Pharmacovigilance Program in a Nutshell}

This program provides the information regarding the ADRs of all the drugs that are available in the country and reports to the Central Drug Regulatory Authority (CDSCO). National Pharmacovigilance Advisory Committee (NPAC) coordinates the program constituted by the Ministry of Health and Family Welfare, Government of India. The program comprises the following steps:

Step 1: to recognize various centers that would provide the ADR-related data

Step 2: health professionals as participants of the national pharmacovigilance program have to be trained and calibrated.

\section{Pharmacovigilance in Dentistry}

The adverse effects of the drugs can be manifested as oral signs and symptoms such as dry mouth, oral ulcerations, taste disturbances, or swellings. Among adverse reactions that occur in 200 most frequently prescribed drugs, dry mouth is very common, constituting $80.5 \%$. This is followed by dysgeusia $(47.5 \%)$ and stomatitis (33.9\%). Evidence was collated from case reports, randomized double-blind controlled studies, nonpeer-reviewed reports, and case series of the oral manifestation of the adverse effect of the drugs. ${ }^{10}$

Efficacy and safety of the drug are main issues to be considered when the drug is administered. It is relatively easier to measure the efficacy of the drug but it may prove to be tedious to measure the safety of the drug. Patients can show different intensities of the adverse effects that occur due to the drug. ${ }^{11}$ Adverse reactions of the drugs are being reported more frequently among hospitalizations. In a systematic review of prospective observational studies, it was estimated that the prevalence of ADR cases reported was $0.16-15.7 \% .^{12}$ The hazards of developing ADRs are growing on one hand, while on the other hand, the identification and reporting standards of ADRs are not adequate. ${ }^{13}$ Geriatric population and medically indigent groups faced the brunt of severe ADRs. ${ }^{14}$
Adverse drug reaction-related international data are found to be meager due to professional attitudes and behavior of the healthcare workers. Hence, for the safer usage of the medicines for the public, there should be appropriate detection, recording, and reporting measures for ADR. A suitably developed pharmacovigilance system might play a critical role in the same. Feely et al. ${ }^{15}$ have reported that reporting of ADRs can be enhanced by offering financial incentives to healthcare workers for every case of ADR that they report. Further studies on methods for strengthening reporting of ADRs among healthcare professionals will shed more light on this aspect of pharmacovigilance.

The pharmacovigilance programs in India are implemented by the government agencies to reduce the frequency of ADRs being reported. There are many ADR monitoring centers in major cities across India established by the Indian Council of Medical Research (ICMR) and Drug Controller General of India. Although there are numerous tertiary care facilities available, the pharmacovigilance system is in its early stages. The major reason may be the minimal understanding and awareness of the pharmacovigilance among healthcare workers in India. In our country, there is underreporting of the cases of ADR, which may be due to the improper training of the staff members or lack of the awareness. Case detection, communication, and the monitoring should be spontaneous among the health workers, which include nurses, dentists, physicians, and pharmacists. The training and methods of enactment should be constantly regulated among different healthcare workers.

Previously reported studies on pharmacovigilance have shown shortcomings in the attitude as well as the knowledge of the healthcare workers pertaining to reporting of ADRs. ${ }^{16-22}$ Every healthcare worker should know where and importantly how to report an ADR. The principles of the pharmacovigilance can be achieved only when there is an active involvement and participation of all the healthcare professionals.

Various studies have explored knowledge, attitudes, and practices of the dental practitioners regarding the ADRs. Iffat et al. ${ }^{23}$ conducted a study in Pakistan, which revealed that many of the dental as well as the medical students felt that reporting the ADR cases to the concerned ministry was important. The respondents also reported that the potential role of the pharmaceutical company was important. In a similar study conducted by Talattof and Azad among dental professionals, authors reported that the dentists had little information regarding the importance, goal, and process of reporting established ADRs. ${ }^{24}$

Praveen et al. ${ }^{25}$ had reported attitude, knowledge, and practice relating to the ADRs among dental and medical practitioners. Authors have observed that there was a significant gap of knowledge in reporting ADRs but favorable attitudes toward reporting ADRs among the participants. Shalini et al. ${ }^{26}$ conducted a similar study on the attitude and knowledge of dental students in a university at Malaysia regarding ADR and pharmacovigilance. Results show that the respondents had low knowledge but reported positive attitude regarding the ADR reporting system and the pharmacovigilance. A study by Bansode et al. ${ }^{27}$ pertaining to the awareness of pharmacovigilance among tertiary care hospitals or resident doctors also revealed low awareness among respondents. To improve the awareness and practices regarding pharmacovigilance, there is an immediate need to train the healthcare professionals.

Arjun et al. ${ }^{28}$ have reported very poor knowledge and poor attitude regarding the pharmacovigilance among the healthcare 
workers in a teaching hospital. Pimpalkhute et al. ${ }^{29}$ have assessed awareness regarding pharmacovigilance and ADR monitoring in tertiary care hospitals with resident doctors with the help of a questionnaire. Authors observed that the lesser numbers of ADRs being reported may be due to poor knowledge regarding the reporting process followed by lack of time due the increased workload in their clinics. Deficient pharmacovigilance training and awareness programs regarding risks of medicines and ADRs at the undergraduate level might also be one of the major impediments.

Khan et al. ${ }^{30}$ explored attitudes, knowledge, and practices of dentists regarding ADRs in a hospital. It was observed that deficiency in attitude and knowledge may be the main reason for low reporting of ADRs by a dentist. A study of attitude regarding pharmacovigilance and ADR reporting was undertaken among healthcare professionals including dentists by Sudhakar et al. ${ }^{31}$ The authors observed that the respondents reported moderate attitude regarding the ADRs without any significant association with gender, experience, and qualification. Gupta et al. ${ }^{19}$ explored the attitude, knowledge, and perceptions of the resident healthcare workers in reporting the ADRs and pharmacovigilance. It was concluded that the awareness regarding the type of event that occurred or the drug that caused the ADR and its reporting mechanisms was found to be deficient. Inadequacy of expertise, lack of sufficient time, poor knowledge regarding the reporting mechanism, and tedious procedures could be the main reasons for the underreporting of the ARDs. ${ }^{32}$ Chhabra et al. ${ }^{33}$ have reported that dental students in Jodhpur, India, had appropriate attitude scores but low knowledge and behavior scores related to pharmacovigilance. In a similar study conducted by Kumar et al., ${ }^{34}$ it was observed that medical and dental students in Wardha, India, had favorable attitude scores. In a systematic review on knowledge, attitude, and practices related to pharmacovigilance among health professionals in India, it was observed that there were many lacunae in knowledge, attitude, and practices among respondents. The authors have highlighted the need for increasing awareness about pharmacovigilance among various health professionals in India. ${ }^{35}$

\section{Challenges that the Pharmacovigilance WiLl Face in Future}

- The information available on the Internet regarding the free availability of drugs may not be entirely reliable. Such information regarding the safety, quality, and efficacy of drugs that are unregistered, prescription drugs, traditional or herbal drugs, and highly controlled substances, may be questionable

- Complications from overdose, interactions, irrational drug use, traditional or herbal medications, polypharmacy, drug abuse, and illegal sale of medications due to the self-medication practice based on the knowledge attained from the Internet, medication error, and also lack of the efficiency of medications all come under the purview of pharmacovigilance

- Imperfections and conflicts in the pharmaceutical industry while dealing with the public's health and their safety in the drug use.

- The generic sector has not fully recognized that it is their responsibility to monitor the safety of the released drug throughout the world

- Understandings of the harm, benefits, and the extent of the acceptable risk regarding the medicines have become less meaningful in the current era of information. In developed and developing countries, the drug-induced diseases have recently been given importance due their increasing morbidity and mortality due to ADRs.

\section{Conclusion}

It can be concluded that pharmacovigilance continues to play a crucial role in meeting the challenges posed by the ever-increasing range and potency of medicines, all of which carry an inevitable and sometimes unpredictable potential for harm. Although health professionals report that paying attention to ADRs and timely reporting is very important, underreporting of ADRs is still a substantial problem. Undergraduate training in pharmacovigilance and awareness about risks associated with medicine use may be either deficient or unsatisfactory to prepare the prospective doctors for the job of ADR monitoring and reporting in their future career.

While prescribing medications in clinical practice, usually a trade-off between potential benefits and harms has to be taken into consideration. The harm that could be caused by medicines can be minimized by improving quality, safety, and efficacy of the medicines and of course rational use. Expectations and concerns of the patient should be taken into consideration when any therapeutic decisions are made. Risks in drug use need to be anticipated and managed effectively. There is a definite need to improve communication between health professionals and the public and educate health professionals to understand the effectiveness or risk of medicines that they prescribe. Pharmacovigilance has definite public health implications as the frequency of ADRs being reported is increasing. ${ }^{36}$ It is essential to foster a sense of trust among patients in the medicines they use, which would eventually pave the way for enhanced confidence in the healthcare delivery system.

\section{References}

1. Fonkwo PN. Pricing infectious disease: the economic and health implications of infectious diseases. EMBO Rep 2008;9(Suppl 1): S13-S17. DOI: 10.1038/embor.2008.110.

2. Brahma DK, Wahlang JB, Marak MD, et al. Adverse drug reactions in the elderly. J Pharmacol Pharmacother 2013;4(2):91-94. DOI: 10.4103/0976-500X.110872.

3. WHO Policy Perspectives on Medicines-Pharmacovigilance: ensuring the safe use of medicines. World Health Organization, Geneva; 2004

4. Agarwal PK An Insight to Pharmacovigilance: A Global Perspective, 1st ed., Mitosis International; 2013. pp. 1-3.

5. Kalaiselvan $V$, Thota P, Singh GN. Pharmacovigilance programme of India: recent developments and future perspectives. Indian J Pharmacol 2016;48(6):624-628. DOI: 10.4103/0253-7613.194855.

6. Suke SG, Kosta P, Negi $\mathrm{H}$. Role of pharmacovigilance in India: an overview. Online J Public Health Inform 2015;7(2):e223. DOI: 10.5210/ ojphi.v7i2.5595.

7. Kalaiselvan V, Srivastava S, Singh A, et al. Pharmacovigilance in India: present scenario and future challenges. Drug Saf 2019;42(3):339-346. DOI: 10.1007/s40264-018-0730-7.

8. National Pharmacovigilance Program [Internet]. 2019 [cited 2019 Mar 15]. Available from: https://www.aiims.edu/en/national-introduction. html?id=166.

9. Bavdekar SB, Karande S. National pharmacovigilance program. Indian Pediatr 2006;43(1):27-32.

10. Smith RG, Burtner AP. Oral side-effects of the most frequently prescribed drugs. Spec Care Dentist 1994;14(3):96-102. DOI: 10.1111/ j.1754-4505.1994.tb01112.x.

11. Hema N, Bhuvana K. Pharmacovigilance: the extent of awareness among the final year students, interns and postgraduates in a government teaching hospital. J Clin Diagn Res 2012;6:1248-1253.

12. Kongkaew C, Noyce PR, Ashcroft DM. Hospital admissions associated with adverse drug reactions: a systematic review of prospective 
observational studies. Ann Pharmacother 2008;42(7):1017-1025. DOI: 10.1345/aph.1L037.

13. Janaje MTM, Singer DR. Costs and prevention of adverse drug reactions. Eur J Intern Med 2001;12(5):403-405. DOI: 10.1016/S09536205(01)00156-X.

14. Wu WK, Pantaleo N. Evaluation of outpatient adverse drug reactions leading to hospitalization. Am J Health Syst Pharm 2003;60(3): 253-259. DOI: 10.1093/ajhp/60.3.253.

15. Feely J, Moriarty $S, O^{\prime}$ Connor $P$. Stimulating reporting of adverse drug reactions by using a fee. BMJ 1990;300(6716):22-23. DOI: 10.1136/ bmj.300.6716.22.

16. Patil A, Gurav YA, Thorat MB, et al. Survey of pharmacovigilance awareness among healthcare professionals. Int J Pharmacol Ther 2014;4:31-34.

17. Gupta SK, Nayak RP, Shivaranjani R, et al. A questionnaire study on the knowledge, attitude, and the practice of pharmacovigilance among the healthcare professionals in a teaching hospital in South India. Perspect Clin Res 2015;6(1):45-52. DOI: 10.4103/2229-3485.148816.

18. Desai CK, lyer G, Panchal J, et al. An evaluation of knowledge, attitude, and practice of adverse drug reaction reporting among prescribers at a tertiary care hospital. Perspect Clin Res 2011;2(4):129-136. DOI: 10.4103/2229-3485.86883.

19. Gupta $P$, Udupa A. Adverse drug reaction reporting and pharmacovigilance: knowledge, attitudes and perceptions amongst resident doctors. J Pharm Sci Res 2011;3:1064-1069.

20. Khan SA, Goyal C, Chandel N, et al. Knowledge, attitudes, and practice of doctors to adverse drug reaction reporting in a teaching hospital in India: an observational study. J Nat Sci Biol Med 2013;4(1):191-196. DOI: 10.4103/0976-9668.107289.

21. Muraraiah S, Rajarathna K, Sreedhar D, et al. A questionnaire study to assess the knowledge, attitude and practice of pharmacovigilance in a paediatric tertiary care centre. J Chem Pharm Res 2011;3:416-422.

22. Remesh A. Identifying the reasons for under reporting of ADR: a cross sectional survey. Res J Pharm Biol Chem Scia 2012;3:1379-1386.

23. Iffat W, Shakeel $S$, Naseem $S$, et al. Attitudinal survey to assess medical and dental students' belief of ADR reporting in Pakistan. Int J Pharm Pharm Sci 2014;6(5):279-283.

24. Talattof Z, Azad A. An evaluation of knowledge, attitude and practice of adverse drug reaction reporting in dental practice. Pak J Nutr 2015;14(10):712-715. DOI: 10.3923/pjn.2015.712.715.

25. Praveen S, Prakash JR, Manjunath GN, et al. Adverse drug reaction reporting among medical and dental practitioners: a KAP study. Indian J Med Spec 2013;4(1):10-15.
26. Shalini S, Mohan S. Knowledge and attitude towards pharmacovigilance and adverse drug reaction reporting among dental students in a private university, Malaysia. J Young Pharm 2015;7(2): 118-125. DOI: 10.5530/jyp.2015.2.10.

27. Bansode AA, Zad VR, Sawant SD, et al. Awareness about pharmacovigilance among resident doctors in a tertiary care hospital. J Evol Med Dent Sci 2015;4(2):207-210. DOI: 10.14260/jemds/ 2015/33.

28. Arjun TN, Sudhir H, Gouraha A, et al. Assessment of knowledge, attitude and practice related to pharmacovigilance among the healthcare professionals in a teaching hospital in central India: an questionnaire study. World J Pharm Sci 2015;4(4):785-799.

29. Pimpalkhute SA, Jaiswal KM, Sontakke SD, et al. Evaluation of awareness about pharmacovigilance and adverse drug reaction monitoring in resident doctors of a tertiary care teaching hospital. Indian J Med Sci 2012;66(3-4):55-61. DOI: 10.4103/0019-5359.110902.

30. Khan SA, Goyal C, Tonpay SD. A study of knowledge, attitudes, and practice of dental doctors about adverse drug reaction reporting in a teaching hospital in India. Perspect Clin Res 2015;6(3):144-149. DOI: 10.4103/2229-3485.159938.

31. Sudhakar S, Madhavan A, Balasubramani S. Attitude of dentists toward pharmacovigilance and reporting adverse drug reactions: a cross-sectional study. J Adv Clin Res Insights 2015;2:242-247. DOI: 10.15713/ins.jcri.87.

32. Ghosh R, Bhatia MS, Bhattacharya SK. Pharmacovigilance: master key to drug safety monitoring and its status in India. Delhi Psychiatry J 2012;15(1):412-415.

33. Chhabra KG, Sharma A, Chhabra C, et al. knowledge, attitude, and practices regarding pharmacovigilance and adverse drug reaction reporting among dental students in a teaching hospital, Jodhpur, India: a cross-sectional Study. J Contemp Dent Pract 2017;18(10): 964-969. DOI: 10.5005/jp-journals-10024-2157.

34. Kumar M, Deolia S, Totade S, et al. Knowledge, attitude and practices of pharmacovigilance among healthcare professionals in a medical and dental institute: a comparative study. Int J Curr Res 2017;9(7):55139-55144.

35. Bhagavathula AS, Elnour AA, Jamshed SQ, et al. Health professionals' knowledge, attitudes and practices about pharmacovigilance in India: a systematic review and meta-analysis. PLoS One 2016;11(3):e0152221. DOI: 10.1371 /journal.pone.0152221.

36. Laporte JR. Fifty years of pharmacovigilance-medicines safety and public health. Pharmacoepidemiol Drug Saf 2016;25(6):725-732. DOI: 10.1002/pds.3967. 